\title{
Effect of screening by external charges on the atomic orbitals and photoinduced processes within the Hartree-Fock-Slater atom
}

\author{
Robert Thiele, ${ }^{1,{ }^{*}}$ Sang-Kil Son, ${ }^{1}$ Beata Ziaja, ${ }^{1,2}$ and Robin Santra ${ }^{1,3}$ \\ ${ }^{1}$ Center for Free-Electron Laser Science, DESY, 2607 Hamburg, Germany \\ ${ }^{2}$ Institute of Nuclear Physics, Polish Academy of Sciences, Radzikowskiego 152, 31-342 Kraków, Poland \\ ${ }^{3}$ Department of Physics, University of Hamburg, 20355 Hamburg, Germany \\ (Received 7 June 2012; published 10 September 2012)
}

\begin{abstract}
X-ray free-electron lasers (XFELs) are a promising tool for the structural determination of macro- and biomolecules, using coherent diffractive imaging. During imaging, the intense XFEL pulses also efficiently ionize the molecules, so it is important to estimate how the charged environment within the molecule modifies atomic properties, in comparison to the case of an isolated atom. Here, we apply the XATOM toolkit to obtain predictions on the modified ionization thresholds and rates of some photoinduced processes in carbon. The Hartree-Fock-Slater model is extended to include the electron screening and ion correlation effects, induced by external charges. With this extended model, we obtain predictions on modifications of orbital energies, photoabsorption cross sections, Auger decay rates, fluorescence emission rates, and atomic scattering factors as a function of the density and temperature of the surrounding charges. Our results have implications for the studies of dynamics within XFEL irradiated samples, in particular for those dedicated to coherent diffraction imaging.

DOI: 10.1103/PhysRevA.86.033411

PACS number(s): 32.90.+a, 32.80.Fb, 87.59.-e, 87.15.ag
\end{abstract}

\section{INTRODUCTION}

Free-electron lasers (FELs) [1-3] provide brilliant radiation with extremely high peak brightness in the soft-X-ray regime (Free-Electron Laser in Hamburg (FLASH) at DESY, Hamburg [4]) or in the hard-X-ray regime (Linac Coherent Light Source (LCLS) in California, SPring-8 Angstrom Compact Free-Electron Laser (SACLA) in Japan, European X-ray Free-Electron Laser (XFEL) [5-7]).

One of the unique opportunities offered by XFELs is single-shot imaging of individual macromolecules [8-10]. Coherent diffraction imaging (CDI) [11] with hard $\mathrm{x}$ rays can determine the structure of noncrystallizing biomolecules or other nanoparticles at atomic resolution [12-25]. The high fluence of a strongly focused XFEL pulse enables the efficient scattering of radiation photons from a molecule, sufficient to obtain a "readable" diffraction pattern of the object. However, during imaging, the intense XFEL pulses also efficiently ionize the molecule. Core-hole states within deep atomic shells are created. This is followed by the relaxation processes such as Auger decay and fluorescence emissions. Secondary electrons released during these processes can further ionize (and damage) the molecule. Therefore, the parameters of the imaging XFEL pulse should be adjusted to the radiation tolerance of the molecule. Simulations of radiation damage enable such adjustment [26-32].

In order to accurately calculate the ionization rates, it is important to know how the charged environment within the molecule modifies the ionization thresholds and rates for the ionization processes, when compared to the case of an isolated atom. This problem belongs to the broad research area of the structure and spectra of atoms confined by various kinds of environments. In recent decades, this area has been attracting the attention of many research groups worldwide, e.g., [33,34].

\footnotetext{
*robert.thiele@cfel.de
}

Here, we treat it in the specific context of coherent diffraction imaging studies that are an important application of XFELs.

In order to estimate this effect, we apply the HartreeFock-Slater (HFS) treatment [35,36], implemented in the computational toolkit XATOM [32]. This code calculates the photoionization cross sections, Auger decay rates, fluorescence rates, and scattering factors in the x-ray regime. In this work, we extend it to include the effect of a charged environment on the photoinduced processes in the context of CDI experiments.

The lowering of the ionization energy within a single atom or ion due to the screening effect was studied by Stewart and Pyatt [37]. More recent calculations were performed by Hilse et al. [38] within a nanoplasma model. Gets and Krainov [39] calculated the ionization potentials for ions in rare-gas clusters, using the Schrödinger equation with the Debye potential included. They found a larger deviation from the isolated-atom case than that given by the Stewart-Pyatt shift. Modifications of atomic properties in a plasma environment have been studied with the Debye screening model and the ion-sphere model for one-electron systems [40-42] and many-electron systems [43-46]. Other studies of the lowering of the ionization energy are summarized in Ref. [47] and, also in the context of electron-ion collision rates, in Refs. [48,49].

In this paper, we present two screening extensions of the HFS model, using the Debye screening model for external thermal electrons and the ion-sphere model for external nonthermal electrons and ions. In the latter, the contribution of surrounding ions is modeled with a radial distribution function. We apply the extended models to calculate orbital energies, photoionization cross sections, and rates for the relaxation processes in carbon, which are relevant for CDI studies of biomolecules.

In such CDI experiments, ions do not form a thermalized plasma during imaging. As the imaging pulses are short (approximately tens of femtoseconds), compared to the electronion equilibration time (approximately a few picoseconds), ions 
remain "cold" during the pulse. Unscreened ions from the outer shell of the irradiated sample can move radially, due to the repulsive Coulomb forces. However, if the pulse is not longer than several femtoseconds, then those unscreened ions will not relocate during the pulse $[29,50]$. As a result, during the imaging pulse, the initially neutral system evolves into a two-regime system consisting of cold ions embedded in a thermalized free-electron plasma.

The paper is organized as follows. In Sec. II, we show how to implement the Debye screening and the ion-sphere model into the HFS treatment. In Sec. III, we calculate the effect of screening for the orbital energies, photoabsorption cross section, and Auger and fluorescence decay rates. We also calculate the modified atomic scattering factors that are necessary for CDI simulations. Finally, in Sec. IV, we conclude with a summary and outlook.

\section{THEORY}

\section{A. Standard Hartree-Fock-Slater model}

For our calculations, we use the XATOM toolkit [32]. The XATOM code is based on the Hartree-Fock-Slater approach [35, 36,51], using the local-density approximation for the exchange interaction. In what follows, atomic units are used, unless specified otherwise. In the HFS approach, the effective singleelectron Schrödinger equation can be written as

$$
\left[-\frac{1}{2} \nabla^{2}+V_{\text {eff }}(\mathbf{r})\right] \psi(\mathbf{r})=\varepsilon \psi(\mathbf{r}),
$$

with the effective potential of the form

$$
V_{\mathrm{eff}}^{\mathrm{HFS}}(\mathbf{r})=-\frac{Z}{r}+\int \frac{\rho\left(\mathbf{r}^{\prime}\right)}{\left|\mathbf{r}-\mathbf{r}^{\prime}\right|} d^{3} r^{\prime}+V_{x}(\mathbf{r}) .
$$

Here, $Z$ is the nuclear charge. The electron density $\rho(\mathbf{r})$ is defined as

$$
\rho(\mathbf{r})=\sum_{i}^{N_{\text {elec }}} \psi_{i}^{\dagger}(\mathbf{r}) \psi_{i}(\mathbf{r})
$$

where the sum extends over all bound electrons, $N_{\text {elec }}$, within the system. The index $i$ denotes the spin-orbital index. The third term in Eq. (2) is the exchange term, which was approximated by Slater [35] as

$$
V_{x}(\mathbf{r})=-\frac{3}{2}\left[\frac{3}{\pi} \rho(\mathbf{r})\right]^{1 / 3} .
$$

In what follows, we will refer to the above equations as the "unscreened case."

\section{B. Debye screening}

We include the Debye screening in the HFS effective potential in the following way:

$$
V_{\mathrm{eff}}^{D}(\mathbf{r})=-\frac{Z e^{-r / \lambda_{D}}}{r}+\int \frac{\rho\left(\mathbf{r}^{\prime}\right) e^{-\left|\mathbf{r}-\mathbf{r}^{\prime}\right| / \lambda_{D}}}{\left|\mathbf{r}-\mathbf{r}^{\prime}\right|} d^{3} r^{\prime}+V_{x}^{D}(\mathbf{r}),
$$

where $\lambda_{D}$ is the Debye screening length. The Debye-screened exchange potential was calculated in Refs. [52,53], and it reads

$$
V_{x}^{D}(\mathbf{r})=V_{x}(\mathbf{r}) F(\alpha),
$$

where $\alpha=1 /\left(\lambda_{D} k_{F}\right)=\kappa_{D} / k_{F}$ is defined as the ratio of the Debye screening parameter $\kappa_{D} \equiv 1 / \lambda_{D}$ and the Fermi momentum $k_{F}$. The correction factor $F(\alpha)$ reads [52]

$$
\begin{aligned}
F(\alpha)= & 1-\frac{\alpha^{2}}{6}-\frac{4}{3} \alpha \tan ^{-1}\left(\frac{2}{\alpha}\right) \\
& +\frac{\alpha^{2}}{2}\left(1+\frac{\alpha^{2}}{12}\right) \ln \left|1+\frac{4}{\alpha^{2}}\right| .
\end{aligned}
$$

As expected, in the limit of $\lambda_{D} \rightarrow \infty$, one arrives at $F(0)=$ 1 , and Eq. (6) reduces to the unscreened (Slater) exchange potential, given by Eq. (4).

The Debye-screened Coulomb potential in Eq. (5) can be expanded in terms of modified Bessel functions and spherical harmonics [54,55] as

$$
\begin{aligned}
\frac{e^{-\left|\mathbf{r}-\mathbf{r}^{\prime}\right| / \lambda_{D}}}{\left|\mathbf{r}-\mathbf{r}^{\prime}\right|}= & \frac{4 \pi}{\sqrt{r r^{\prime}}} \sum_{l=0}^{\infty} I_{l+\frac{1}{2}}\left(\frac{r_{<}}{\lambda_{D}}\right) K_{l+\frac{1}{2}}\left(\frac{r_{>}}{\lambda_{D}}\right) \\
& \times \sum_{m=-l}^{l} Y_{l m}(\theta, \phi) Y_{l m}^{*}\left(\theta^{\prime}, \phi^{\prime}\right),
\end{aligned}
$$

where $I_{l+\frac{1}{2}}(x)$ and $K_{l+\frac{1}{2}}(x)$ are the modified Bessel functions, $r_{<}$is $\min \left(r, r^{\prime}\right), r_{>}$is $\max \left(r, r^{\prime}\right)$, and $Y_{l m}(\theta, \phi)$ are the spherical harmonics. Assuming a spherically symmetric density of bound electrons, $\rho(\mathbf{r}) \rightarrow \rho(r)$, the integral of the spherical harmonics over $\theta^{\prime}$ and $\phi^{\prime}$ in Eq. (5) is zero except for the case of $l=0$ and $m=0$, and then the second term in Eq. (5) yields

$$
\begin{aligned}
& \int \frac{\rho\left(r^{\prime}\right) e^{-\left|\mathbf{r}-\mathbf{r}^{\prime}\right| / \lambda_{D}}}{\left|\mathbf{r}-\mathbf{r}^{\prime}\right|} d^{3} r^{\prime} \\
& =4 \pi \int \frac{\rho\left(r^{\prime}\right)}{\sqrt{r r^{\prime}}} I_{\frac{1}{2}}\left(\frac{r_{<}}{\lambda_{D}}\right) K_{\frac{1}{2}}\left(\frac{r_{>}}{\lambda_{D}}\right) r^{\prime 2} d r^{\prime} \\
& =4 \pi \int_{0}^{\infty} \frac{\lambda_{D} \rho\left(r^{\prime}\right)}{2 r r^{\prime}}\left[e^{-\frac{r_{>}-r_{<}}{\lambda_{D}}}-e^{-\frac{r_{>}+r_{<}}{\lambda_{D}}}\right] r^{\prime 2} d r^{\prime} .
\end{aligned}
$$

This simplified expression is then evaluated numerically by the XATOM code.

\section{Ion-sphere model}

The previous screening model takes into account only the screening effect of thermalized free electrons in the weakcoupling regime. Now we also account for the presence of external ions, using the ion-sphere model $[40,47]$ with constant electron and ion densities. The net charge is assumed to be equal to zero. The ion-sphere HFS potential can be written as

$$
V_{\mathrm{eff}}^{\mathrm{IS}}(\mathbf{r})=-\frac{Z}{r}+\int \frac{\rho\left(\mathbf{r}^{\prime}\right)+\bar{n}_{e}-\bar{Z}_{i} \bar{n}_{i} g_{i i}\left(r^{\prime}\right)}{\left|\mathbf{r}-\mathbf{r}^{\prime}\right|} d^{3} r^{\prime}+V_{x}(\mathbf{r}),
$$

where $\bar{n}_{e}$ and $\bar{n}_{i}$ are the number densities of external electrons and ions, respectively. The charge $\bar{Z}_{i}$ is the effective charge of ions. In contrast to conventional ion-sphere models [40,47], we introduce the ion-ion radial distribution function $g_{i i}(r)$ [56], assuming our system to be isotropic and homogeneous. Further, the assumed quasineutrality of the system implies $\bar{Z}_{i} \bar{n}_{i}-\bar{n}_{e}=0$. 


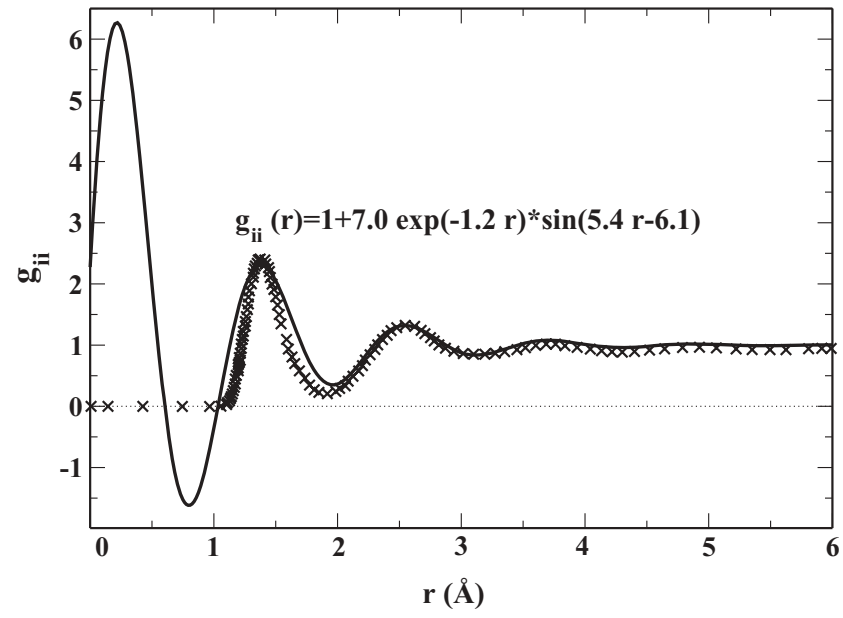

FIG. 1. Ion-ion radial distribution function for liquid carbon with a density of $2.0 \mathrm{~g} / \mathrm{cm}^{3}$. Comparison between the MD calculation by Wang et al. [59] (crosses) and the fitting formula by Broyles et al. [57,58] (solid line). The fitted parameters are $a=7.0, b=$ $1.2 \AA^{-1}, c=5.4 \AA^{-1}$, and $d=6.1$.

\section{Radial distribution function}

In order to parametrize $g_{i i}(r)$, we use the fitting formula proposed by Broyles et al. [57,58],

$$
g_{i i}(r)=1+a e^{-b r} \sin (c r-d),
$$

with four fit parameters, $a, b, c, d$. The parameters $a$ and $d$ are unitless, and the parameters $b$ and $c$ have the unit of $\AA^{-1}$.

For the calculation of the fit parameters in Eq. (11), we employed the results of the molecular dynamics (MD) calculation by Wang et al. [59]. These calculations were performed for liquid carbon (of density $2.0 \mathrm{~g} / \mathrm{cm}^{3}$ ) and compressed liquid carbon (of density $4.4 \mathrm{~g} / \mathrm{cm}^{3}$ ). They are shown in Figs. 1 and 2. For both (liquid and compressed) carbon cases, our fits are in good agreement with the MD calculations. Let us remark that the negative values of the

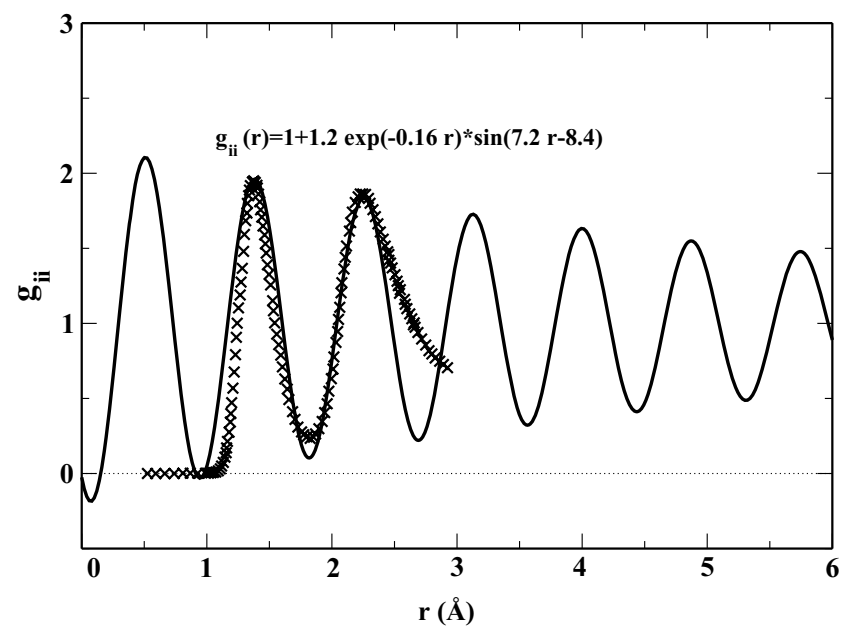

FIG. 2. Ion-ion radial distribution function for liquid carbon with a density of $4.4 \mathrm{~g} / \mathrm{cm}^{3}$. Comparison between the MD calculation by Wang et al. [59] (crosses) and the fitting formula by Broyles et al. [57,58] (solid line). The fitted parameters are $a=1.2, b=$ $0.16 \AA^{-1}, c=7.2 \AA^{-1}$, and $d=8.4$. fitting formula are not physical. Therefore, when applying the fit to XATOM calculations, we set $g_{i i}(r)=0$ at $r<r_{c}$ and apply the fit for $r \geqslant r_{c}$, where $r_{c}$ is the largest zero of Eq. (11). This simple fitting formula can then be easily implemented in the XATOM code, allowing for the efficient numerical integration of Eq. (10).

Let us note that these MD simulations were performed for the neutral carbon liquid. They can be applied to an ionized carbon system only if the internal Coulomb interaction does not displace the ions. This assumption is valid in the regime relevant for CDI simulations, since ion positions are effectively frozen during an ultrashort x-ray pulse. For an ionized plasma with charges in thermal equilibrium, a semiempirical correlation function from [60] should be used instead.

\section{RESULTS}

In this section, we calculate the screening effect by electrons (Debye model) and by both electrons and ions (ion-sphere model) on the orbital energies, photoabsorption cross sections, Auger and fluorescence rates, and atomic scattering factors. Within this extended HFS approach, the Debye length $\lambda_{D}$ and the properties of the ion environment (the average ion charge $\bar{Z}_{i}$ and the ion number density $\bar{n}_{i}$ ) are free parameters that we define in the model. In what follows, we will apply the parameter range relevant for CDI simulations (cf. [61]). Correspondingly, the density of liquid carbon $\left(2.0 \mathrm{~g} / \mathrm{cm}^{3}\right)$ is close to that of organic molecules. The case of compressed carbon $\left(4.4 \mathrm{~g} / \mathrm{cm}^{3}\right)$ is considered in order to evaluate the impact of high ion density on the model results (strong screening regime).

\section{A. Orbital energy}

In Fig. 3, we show the energy shifts between the orbitals determined from the unscreened HFS model and the Debye

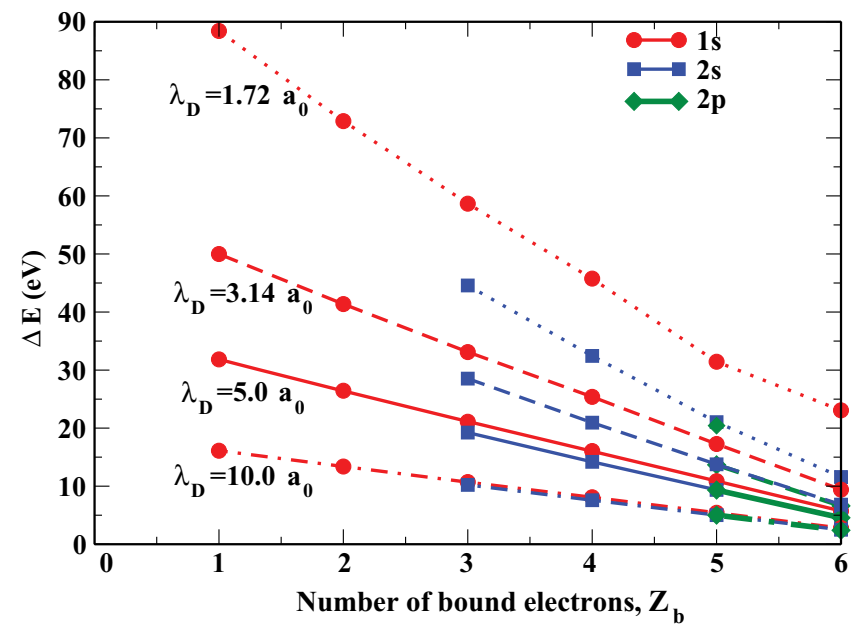

FIG. 3. (Color online) Energy shifts $\Delta E$ between the unscreened HFS model and the Debye-screened HFS model for carbon as a function of the number of the electrons bound on carbon, $Z_{b}$. The calculations were performed for several Debye screening lengths $\left(\lambda_{D}=1.72,3.14,5.0,10.0 a_{0}\right)$. Shifts for the various orbitals $1 s, 2 s$, and $2 p$ are shown. 


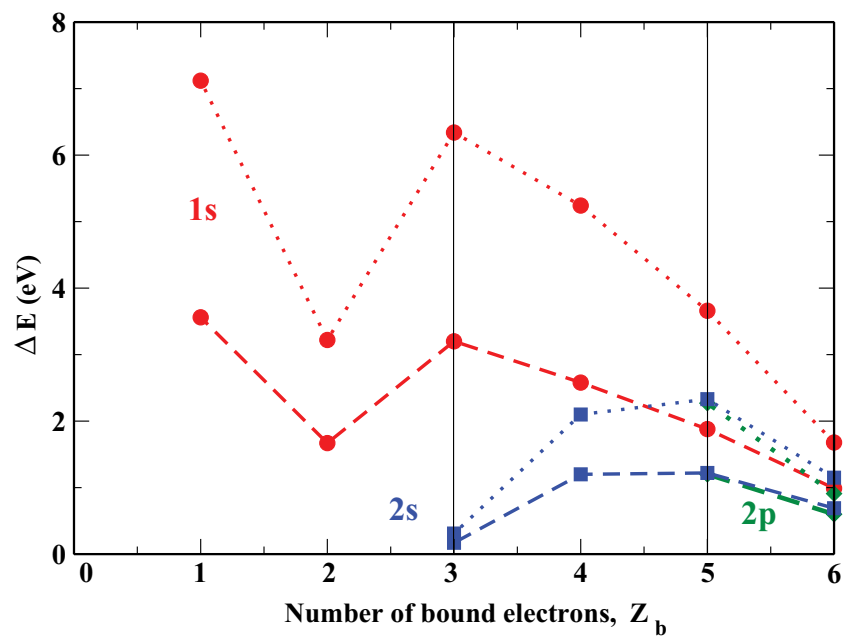

FIG. 4. (Color online) Energy shifts $\Delta E$ between the unscreened HFS model and the ion-sphere screened HFS model for carbon as a function of the number of the electrons bound on carbon, $Z_{b}$. The external ion density is $2.0 \mathrm{~g} / \mathrm{cm}^{3}$. External ions have charge $\bar{Z}_{i}=1$ (dashed lines) and $\bar{Z}_{i}=2$ (dotted lines), respectively.

screened HFS model, given by Eq. (5), as a function of the number of electrons $Z_{b}$ bound to the carbon atom or ion, i.e., $Z_{b}=6$ for $\mathrm{C}^{0+}, Z_{b}=5$ for $\mathrm{C}^{1+}$, etc. These shifts are approximately linear functions of the number of bound electrons. They increase with the decreasing screening length and are largest for the inner-shell orbital $(1 s)$. The screening does not "distinguish" between orbitals of the same principal quantum number $(2 s, 2 p)$ : the calculated shifts are almost identical in this case. We have checked that at $\lambda_{D} \rightarrow \infty$, the energy shift $\Delta E=0$, and the screened case reduces to the unscreened case, as expected. Let us note that at decreasing screening length, the orbital energies approach the continuum. At some limiting values of $\lambda_{D}$, the energy of the uppermost filled orbital will overlap with the continuum. This defines the limit of applicability of our model. For $\lambda_{D}=1.72 a_{0}\left(a_{0}\right.$ is the Bohr radius), the $2 p$-orbital energy of $\mathrm{C}^{0+}$ becomes positive. The same behavior of increasing the orbital energy up to a positive value in a Debye-screened environment was observed in $[42,43]$.

In Figs. 4 and 5, we show the energy shifts for different orbitals obtained within the ion-sphere screened HFS model compared to those obtained in the unscreened case. For these calculations, we used the radial distribution functions fitted for ion densities of $2.0 \mathrm{~g} / \mathrm{cm}^{3}$ and $4.4 \mathrm{~g} / \mathrm{cm}^{3}$; see Figs. 1 and 2 . In contrast to the Debye-screened HFS model, the calculated shifts are nonlinear functions of the number of bound electrons. The curves show characteristic minima that occur after the preceding shell was emptied of electrons. Again, the lowerlying orbital $(1 s)$ is more affected by screening than the upper ones $(2 s, 2 p)$, and the screening does not distinguish between the orbitals of the same principal quantum number. As expected, the effect of screening becomes stronger for a more highly charged environment: energy shifts for the external ion charge $\bar{Z}_{i}=2$ are larger than those for $\bar{Z}_{i}=1$.

In Fig. 6, we show the orbital energies for the $1 s$ and $2 s$ shells of $\mathrm{C}^{3+}$ ion in the ground configuration. Accordingly, the $2 p$ shell is not occupied. The orbital energy depends approx-

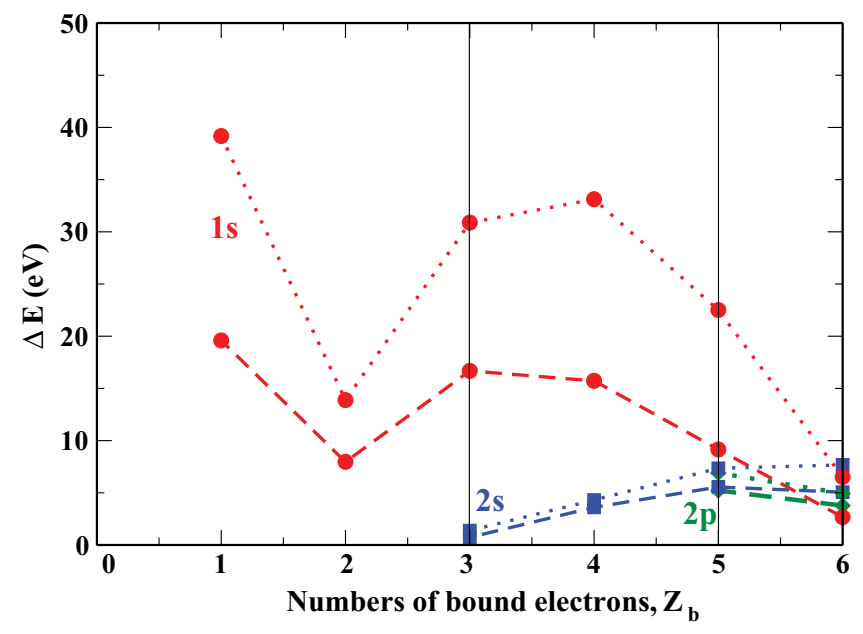

FIG. 5. (Color online) Energy shifts $\Delta E$ between the unscreened HFS model and the ion-sphere screened HFS model for carbon as a function of the number of the electrons bound on carbon, $Z_{b}$. The external ion density is $4.4 \mathrm{~g} / \mathrm{cm}^{3}$. External ions have charge $\bar{Z}_{i}=1$ (dashed lines) and $\bar{Z}_{i}=2$ (dotted lines), respectively.

imately linearly [cf. Eq. (10)] on the charge of surrounding ions, $\bar{Z}_{i}$. As expected, this effect is stronger for the denser ion environment.

Figure 7 shows the radial wave functions of the $1 s$ (red line), $2 s$ (blue line), and $2 p$ (green line) subshells of neutral carbon. The $y$ axis is $\left|P_{n l}(r)\right|^{2}$, where $P_{n l}(r)$ is the radial wave function. It compares the unscreened (solid line), Debyescreened (dashed line, $\lambda_{D}=5 a_{0}$ ), and ion-sphere (dotted line, $\left.2.0 \mathrm{~g} / \mathrm{cm}^{2}, \bar{Z}_{i}=2\right)$ models. Even though the inner-shell $(1 \mathrm{~s})$ orbital energy is more shifted than the energy of other orbitals, the shape of the $1 s$ orbital is barely changed when the two screening models are applied. In contrast, the shapes of $2 s$ and $2 p$ for both screening models deviate from the unscreened case, implying that the valence orbitals are delocalized due

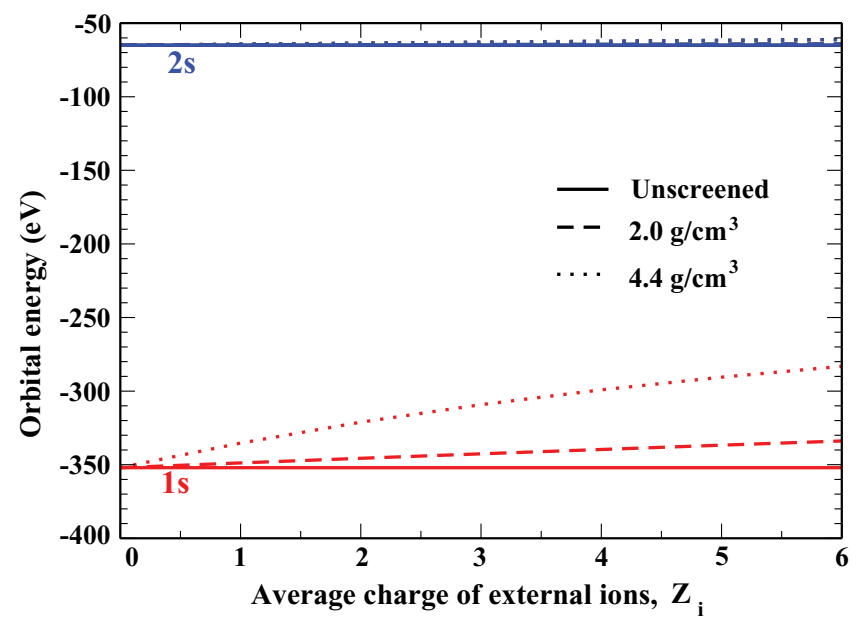

FIG. 6. (Color online) Orbital energy for the $1 s$ (red lines) and $2 s$ (blue lines) shells for $\mathrm{C}^{3+}$ in the ground state as a function of the average charge of external ions, $\bar{Z}_{i}$. The solid line shows the result of the unscreened HFS model. The dashed and the dotted lines show the ion-sphere screened results for the carbon densities of $2.0 \mathrm{~g} / \mathrm{cm}^{3}$ and $4.4 \mathrm{~g} / \mathrm{cm}^{3}$, respectively. 


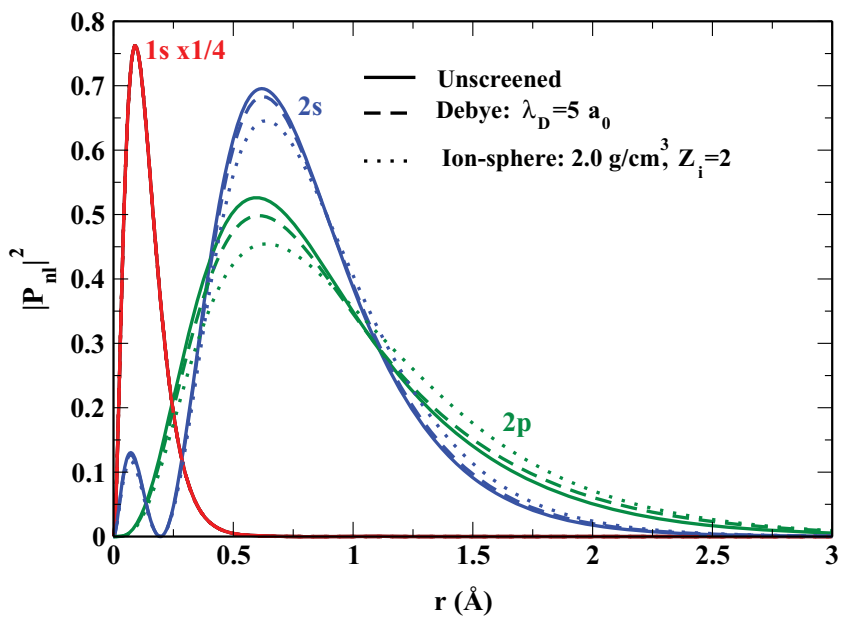

FIG. 7. (Color online) Comparison of $1 s, 2 s$, and $2 p$ radial wave functions of neutral carbon for the unscreened, Debye-screened, and ion-sphere models. For the Debye-screened model, $\lambda_{D}=5 a_{0}$ is used, and for the ion-sphere model, the ion density of $2.0 \mathrm{~g} / \mathrm{cm}^{3}$ and $\bar{Z}_{i}=2$ are used.

to the screening effect. The atomic potential for bound electrons is weakened by screening from free electrons, and bound electrons are pulled out due to the existence of neighboring ions. The valence electrons are more affected by those screening effects than the core electrons. We emphasize here that although the plots of the screening-induced absolute energy shifts, $\Delta E$, show the largest energy shifts for core electrons, the respective relative shifts calculated in respect to the unscreened energy level are smallest for the deep-lying orbitals.

\section{B. Photoabsorption cross section}

The effect of screening on the photoabsorption cross section is shown in Fig. 8. Here we compare the total photoabsorption cross section for carbon in the ground state, calculated with different models. The reference is the result obtained from the unscreened HFS model. Debye screening shifts the ionization threshold. As expected, for $\lambda_{D}=10.0 a_{0}$, this shift is correspondingly smaller than for $\lambda_{D}=5.0 a_{0}$. The Debye-screened cross section is "extrapolated" towards the shifted threshold (cf. Fig. 9 for Debye-screened photoabsorption from different shells) without much affecting the cross-section magnitude. In the case of ion-sphere screening, the thresholds are shifted towards the "screened" value, but the magnitude of the cross section also increases in the vicinity of the threshold. Again, this effect is more pronounced in the case of the denser ion environment. The important observation is that the screening effects manifest themselves in the cross section only at lower photon energies (VUV, soft x-ray). The hard-x-ray regime seems to be unaffected by the screening. This is significant for CDI simulations, as it may justify using the unscreened cross sections in such simulations.

\section{Auger and fluorescence processes}

Auger and fluorescence effects are relaxation processes of an inner-shell vacancy within an atom. Photoabsorption at

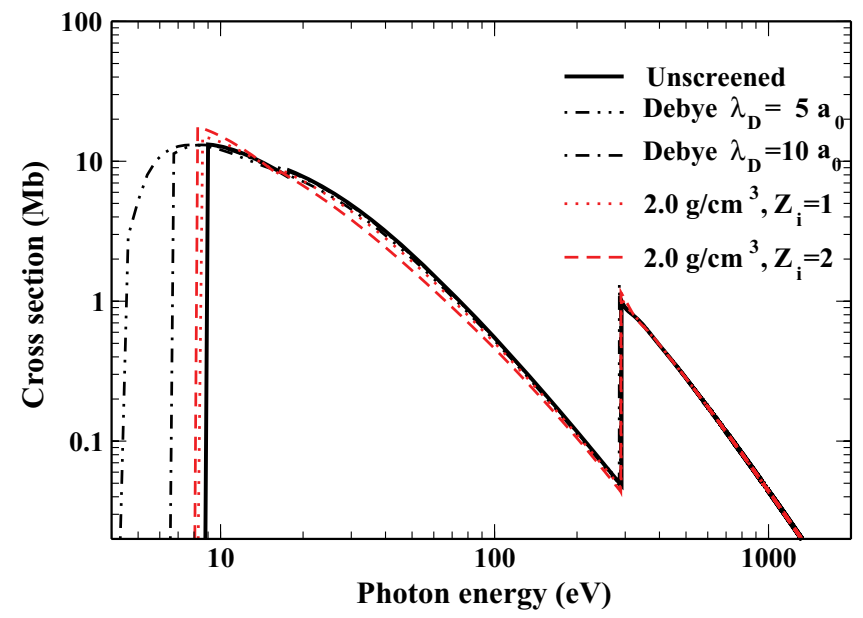

FIG. 8. (Color online) Total photoabsorption cross section for carbon in the ground state calculated with XАTOM as a function of the photon energy for (i) the unscreened HFS model (black solid line), (ii) the Debye-screened HFS model $\left(\lambda_{D}=5.0 a_{0}\right.$, black doubledot-dashed line; $\lambda_{D}=10 a_{0}$, black dot-dashed line), and (iii) the ion-sphere screened HFS model (density of $2.0 \mathrm{~g} / \mathrm{cm}^{3}, \bar{Z}_{i}=1,2$, red dashed line and red dotted line).

high x-ray energy typically leads to the creation of such core holes. The relaxation processes are then an important part of the electronic damage. An accurate calculation of their rates is necessary for preparing a reliable CDI simulation.

In Tables I and II, we show the Auger and fluorescence rates calculated for the Debye-screened and the ion-sphere screened HFS models. They are compared to the unscreened HFS calculations [32]. All possible electronic configurations of carbon for a given charge state are considered. For the Auger rate calculation, the Debye screening effect is only incorporated through the screened orbitals. Thus, the same expression for the Auger rate as in Ref. [32] is employed.

For the Debye calculation in Table I, we have chosen the Debye screening length of $\lambda_{D}=5.0 a_{0}$. The screening

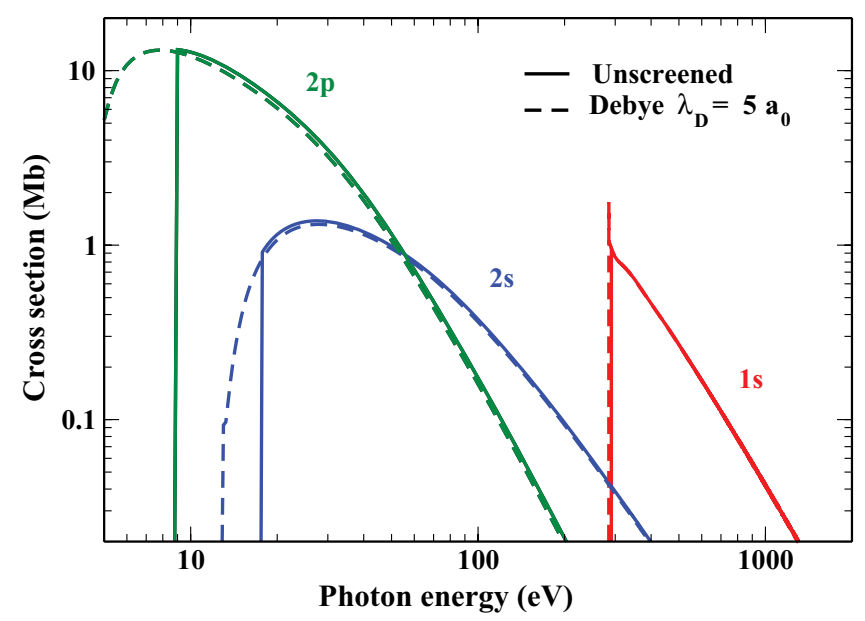

FIG. 9. (Color online) Subshell photoabsorption cross section for carbon in the ground state for different shells calculated with XATOM for the unscreened HFS model (solid lines) and for the Debye-screened HFS model ( $\lambda_{D}=5.0 a_{0}$, dashed lines) as a function of the photon energy. 
TABLE I. Auger rates $\left(\Gamma_{A}\right)$ and fluorescence rates $\left(\Gamma_{F}\right)$ calculated with the Debye-screened HFS model for the Debye length, $\lambda_{D}=5.0 a_{0}$, for various electron configurations of carbon. For better comparison, the unscreened HFS results by Son et al. [32] are shown in parentheses.

\begin{tabular}{|c|c|c|c|c|c|}
\hline \multirow[b]{2}{*}{ Charge } & \multirow[b]{2}{*}{ Configuration } & \multicolumn{3}{|c|}{$\Gamma_{A}\left(10^{-3}\right.$ a.u. $)$} & \multirow{2}{*}{$\frac{\Gamma_{F}\left(10^{-5} \text { a.u. }\right)}{K \alpha}$} \\
\hline & & $K L_{1} L_{1}$ & $K L_{1} L_{23}$ & $K L_{23} L_{23}$ & \\
\hline+1 & $1 s^{1} 2 s^{2} 2 p^{2}$ & $0.918(0.961)$ & $0.914(0.970)$ & $0.408(0.439)$ & $0.801(0.836)$ \\
\hline \multirow[t]{3}{*}{+2} & $1 s^{0} 2 s^{2} 2 p^{2}$ & $2.781(2.894)$ & $3.197(3.333)$ & $1.677(1.755)$ & $2.663(2.740)$ \\
\hline & $1 s^{1} 2 s^{1} 2 p^{2}$ & & $0.572(0.602)$ & $0.539(0.574)$ & $0.938(0.975)$ \\
\hline & $1 s^{1} 2 s^{2} 2 p^{1}$ & $1.124(1.178)$ & $0.587(0.620)$ & & $0.479(0.498)$ \\
\hline \multirow[t]{5}{*}{+3} & $1 s^{0} 2 s^{1} 2 p^{2}$ & & $1.901(1.978)$ & $2.043(2.128)$ & $2.957(3.041)$ \\
\hline & $1 s^{0} 2 s^{2} 2 p^{1}$ & $3.333(3.462)$ & 1.918 (1.994) & & 1.507 (1.549) \\
\hline & $1 s^{1} 2 s^{0} 2 p^{2}$ & & & $0.665(0.703)$ & $1.070(1.108)$ \\
\hline & $1 s^{1} 2 s^{1} 2 p^{1}$ & & $0.353(0.370)$ & & $0.549(0.569)$ \\
\hline & $1 s^{1} 2 s^{2} 2 p^{0}$ & $1.336(1.394)$ & & & \\
\hline \multirow[t]{4}{*}{+4} & $1 s^{0} 2 s^{0} 2 p^{2}$ & & & $2.488(2.586)$ & $3.191(3.273)$ \\
\hline & $1 s^{0} 2 s^{1} 2 p^{1}$ & & $1.108(1.148)$ & & $1.651(1.694)$ \\
\hline & $1 s^{0} 2 s^{2} 2 p^{0}$ & $3.789(3.915)$ & & & \\
\hline & $1 s^{1} 2 s^{0} 2 p^{1}$ & & & & $0.623(0.648)$ \\
\hline+5 & $1 s^{0} 2 s^{0} 2 p^{1}$ & & & & 1.915 (1.965) \\
\hline
\end{tabular}

by free electrons lowers the calculated relaxation rates. The differences between the screened and unscreened models are smaller than 5\%. This screening-induced damping of relaxation rates significantly increases with decreasing Debye length by up to $10 \%$ for $\lambda_{D}=3.14 a_{0}$ and up to $30 \%$ for $\lambda_{D}=1.72 a_{0}$ (not shown).

In the case of the ion-sphere screened HFS model, the rates are also reduced when the screening is applied (Table II). For the ion density of $2.0 \mathrm{~g} / \mathrm{cm}^{3}$ and the average ion charge of $\bar{Z}_{i}=2$, the differences with the unscreened model reach up to $15 \%$ for some electron configurations. These differences further increase with increasing ionization state $\bar{Z}_{i}$ and increasing ion density, up to $65 \%$ for the ion density of $4.4 \mathrm{~g} / \mathrm{cm}^{3}$ and $\bar{Z}_{i}=2$.

The screening-induced reduction of relaxation rates can be explained as follows. As shown in Fig. 7, the valence orbitals tend to be delocalized due to the screening effect. The fluorescence rate from $2 p$ to $1 s$ contains the transition dipole matrix element between them. The Auger rate expression involves the Coulomb matrix elements among core, valence, and continuum states. When the valence orbitals become more diffuse relative to the core orbitals, those matrix elements will be reduced. Also, the screening-induced fluorescence rate is affected by the differences in energy shifts between core and valence orbitals, as discussed in Sec. III A, since this rate is proportional to the cube of the transition energy between $1 \mathrm{~s}$ and $2 p$ orbitals. As a result, the fluorescence and Auger rates are decreased when screening is applied.

It is worthwhile to note that this decrement of rates might be overestimated within the current screening model based on the atomic system because the current model does not take into account the contribution to the atomic potential

TABLE II. Auger rates $\left(\Gamma_{A}\right)$ and fluorescence rates $\left(\Gamma_{F}\right)$ calculated with the ion-sphere screened HFS model for various electronic configurations of carbon with ion density of $2.0 \mathrm{~g} / \mathrm{cm}^{3}$ and average charge of $\bar{Z}_{i}=2$. For better comparison, the unscreened HFS results by Son et al. [32] are shown in parentheses.

\begin{tabular}{|c|c|c|c|c|c|}
\hline \multirow[b]{2}{*}{ Charge } & \multirow[b]{2}{*}{ Configuration } & \multicolumn{3}{|c|}{$\Gamma_{A}\left(10^{-3}\right.$ a.u. $)$} & \multirow{2}{*}{$\frac{\Gamma_{F}\left(10^{-5} \text { a.u. }\right)}{K \alpha}$} \\
\hline & & $K L_{1} L_{1}$ & $K L_{1} L_{23}$ & $K L_{23} L_{23}$ & \\
\hline+1 & $1 s^{1} 2 s^{2} 2 p^{2}$ & $0.832(0.961)$ & $0.821(0.970)$ & $0.366(0.439)$ & $0.761(0.836)$ \\
\hline \multirow[t]{3}{*}{+2} & $1 s^{0} 2 s^{2} 2 p^{2}$ & $2.583(2.894)$ & $2.978(3.333)$ & $1.568(1.755)$ & $2.586(2.740)$ \\
\hline & $1 s^{1} 2 s^{1} 2 p^{2}$ & & $0.513(0.602)$ & $0.478(0.574)$ & $0.890(0.975)$ \\
\hline & $1 s^{1} 2 s^{2} 2 p^{1}$ & $1.012(1.178)$ & $0.528(0.620)$ & & $0.455(0.498)$ \\
\hline \multirow{5}{*}{+3} & $1 s^{0} 2 s^{1} 2 p^{2}$ & & $1.764(1.978)$ & $1.889(2.128)$ & $2.852(3.041)$ \\
\hline & $1 s^{0} 2 s^{2} 2 p^{1}$ & $3.069(3.462)$ & 1.789 (1.994) & & 1.457 (1.549) \\
\hline & $1 s^{1} 2 s^{0} 2 p^{2}$ & & & $0.599(0.703)$ & 1.008 (1.108) \\
\hline & $1 s^{1} 2 s^{1} 2 p^{1}$ & & $0.316(0.370)$ & & $0.516(0.569)$ \\
\hline & $1 s^{1} 2 s^{2} 2 p^{0}$ & $1.201(1.394)$ & & & \\
\hline \multirow[t]{4}{*}{+4} & $1 s^{0} 2 s^{0} 2 p^{2}$ & & & $2.321(2.586)$ & $3.050(3.273)$ \\
\hline & $1 s^{0} 2 s^{1} 2 p^{1}$ & & $1.027(1.148)$ & & 1.577 (1.694) \\
\hline & $1 s^{0} 2 s^{2} 2 p^{0}$ & $3.505(3.915)$ & & & \\
\hline & $1 s^{1} 2 s^{0} 2 p^{1}$ & & & & $0.622(0.648)$ \\
\hline+5 & $1 s^{0} 2 s^{0} 2 p^{1}$ & & & & $1.911(1.965)$ \\
\hline
\end{tabular}




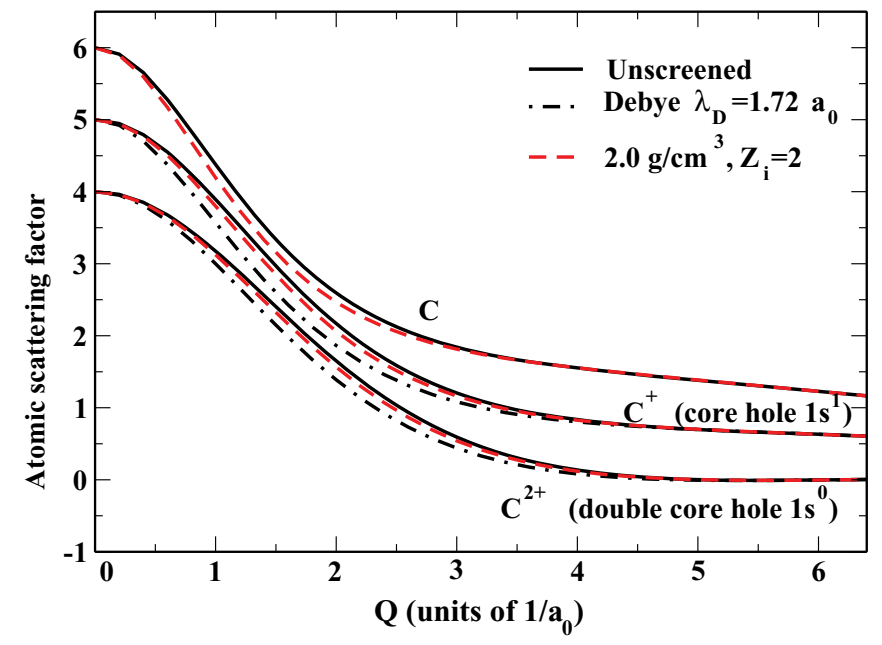

FIG. 10. (Color online) Atomic scattering factor of carbon for different electronic configurations calculated as a function of the momentum transfer. We show the unscreened HFS case (black solid line), the Debye-screened model for $\lambda_{D}=1.72 a_{0}$ (black dot-dashed line), and the ion-sphere screened HFS model with the density of $2.0 \mathrm{~g} / \mathrm{cm}^{3}, \bar{Z}_{i}=2$ (red dashed line).

from delocalized electrons of neighboring atoms. For a more accurate treatment of delocalization effects, molecular calculations would be required.

\section{Atomic scattering factor}

Assuming spherically symmetric density of the bound electrons, one can calculate the atomic scattering factor as

$$
f(Q)=4 \pi \int_{0}^{\infty} r^{2} \rho(r) \frac{\sin (Q r)}{Q r} d r,
$$

where $Q$ is the magnitude of the photon momentum transfer. The atomic scattering factors calculated for carbon are shown in Fig. 10. We considered three different configurations: atomic carbon, $C^{1+}$ ion with a core hole in the $1 s$ shell, and $C^{2+}$ ion with a double core hole in the $1 s$ shell. The results from the two screening models are compared to the unscreened HFS case. The differences between the unscreened and screened scattering factors for the considered parameters $\left(\lambda_{D}=1.72\right.$ $a_{0}$ for the Debye model, and the density of $2.0 \mathrm{~g} / \mathrm{cm}^{3}, \bar{Z}_{i}=2$ for the ion-sphere model) are less than $10 \%$. Again, this finding is significant for CDI simulations, as even by using the unscreened model to calculate the scattering factors, one can obtain predictions of sufficient accuracy. Let us note that all models fulfill the forward-scattering sum rule at $Q=0, f(Q)=Z_{b}$. The differences between the models show up in the region of $0.5<Q<4 a_{0}^{-1}$. For larger $Q$ values, the predictions for the screened and unscreened cases again converge.

\section{CONCLUSIONS}

In this paper, we extended the standard HFS model and its numerical implementation within the XATOM code in order to include screening effects by free electrons and external ions, present in the vicinity of the central atom. We considered (i) the Debye-screened model, accounting for screening by thermalized free electrons, and (ii) the ion-sphere model, accounting for screening by nonthermalized electrons and ions in an asymptotically net-neutral system. The contribution of external ions was modeled with a radial distribution function that was fitted in order to match the corresponding MD calculations.

The charged environment of the central atom entered the extended HFS model through free parameters: the Debye length for the Debye-screened model, and the density and average charge of external ions for the ion-sphere screened model. For the latter, the external electron density was fixed by the quasineutrality condition.

Our analysis focused on the parameter regime corresponding to the dynamics within XFEL irradiated samples, in particular on the regime relevant for CDI studies. Therefore, we have obtained predictions for various configurations of carbon atoms, which constitute a typical object for CDI studies.

First, we calculated the orbital energies for carbon within the two screened HFS models and compared them to the unscreened case. Energies obtained with both models were shifted towards the continuum, lowering the ionization thresholds. At strong screening (short Debye screening length or dense ion-electron surrounding), outer orbitals overlap with the continuum and the corresponding electrons become ionized.

Screening-induced changes in photoabsorption cross sections manifested themselves only in the low photon energy regime. The hard-x-ray regime was almost unaffected. Atomic scattering factors were also weakly influenced by the screening effects. In contrast, for the Auger and fluorescence rates, screening always resulted in a strong reduction of those rates. This effect was due to the spatial delocalization of valence orbitals induced by the screening.

Our calculations show that the XATOM code with the implemented extension for the Debye and the ion-sphere screening is a promising attempt to include the effects of a charged plasma environment in atomic calculations. At present, the screening environment enters the HFS approach through free parameters. In the future, we plan to extend our study to include a self-consistent adjustment of these parameters during the system evolution.

\section{ACKNOWLEDGMENTS}

We thank Zoltan Jurek, Nikita Medvedev and Mohamed El-Amine Madjet for helpful discussions. We thank Otfried Geffert for programming support.
[1] J. Feldhaus, J. Arthur, and J. B. Hastings, J. Phys. B 38, 799 (2005).

[2] C. Pellegrini and S. Reiche, J. Sel. Top. Quantum Electron. 10, 1393 (2004).
[3] B. W. J. McNeil and N. R. Thompson, Nature Photon. 4, 814 (2010).

[4] W. Ackermann et al., Nature Photon. 1, 336 (2007).

[5] P. Emma et al., Nature Photon. 4, 641 (2010). 
[6] D. Pile, Nature Photon. 5, 456 (2011).

[7] M. Altarelli et al., The European X-Ray Free-Electron Laser Technical Design Report (DESY, Germany, 2007).

[8] J. Hajdu, Curr. Opin. Struct. Biol. 10, 569 (2000).

[9] K. J. Gaffney and H. N. Chapman, Science 316, 1444 (2007).

[10] J. Miao, T. Ishikawa, Q. Shen, and T. Earnest, Annu. Rev. Phys. Chem. 59, 387 (2008).

[11] H. N. Chapman and K. A. Nugent, Nature Photon. 4, 833 (2010).

[12] J. Miao, P. Charalambous, J. Kirz, and D. Sayre, Nature (London) 400, 342 (1999).

[13] I. K. Robinson, I. A. Vartanyants, G. J. Williams, M. A. Pfeifer, and J. A. Pitney, Phys. Rev. Lett. 87, 195505 (2001).

[14] H. N. Chapman et al., Nature Phys. 2, 839 (2006).

[15] H. N. Chapman et al., Nature (London) 448, 676 (2007).

[16] K. E. Schmidt, J. C. H. Spence, U. Weierstall, R. Kirian, X. Wang, D. Starodub, H. N. Chapman, M. R. Howells, and R. B. Doak, Phys. Rev. Lett. 101, 115507 (2008).

[17] A. Barty, S. Boutet, M. J. Bogan, S. Hau-Riege, S. Marchesini, K. Sokolowski-Tinten, N. Stojanovic, R. Tobey, H. Ehrke, A. Cavalleri, S. Düsterer, M. Frank, S. Bajt, B. W. Woods, M. M. Seibert, J. Hajdu, R. Treusch, and H. N. Chapman, Nature Photon. 2, 415 (2008).

[18] S. Marchesini, S. Boutet, A. E. Sakdinawat, M. J. Bogan, S. Bajt, A. Barty, H. N. Chapman, M. Frank, S. P. Hau-Riege, A. Szöke, C. Cui, D. A. Shapiro, M. R. Howells, J. C. H. Spence, J. W. Shaevitz, J. Y. Lee, J. Hajdu, and M. M. Seibert, Nature Photon. 2, 560 (2008).

[19] M. J. Bogan, W. H. Benner, S. Boutet, U. Rohner, M. Frank, A. Barty, M. M. Seibert, F. Maia, S. Marchesini, S. Bajt, B. Woods, V. Riot, S. P. Hau-Riege, M. Svenda, E. Marklund, E. Spiller, J. Hajdu, and H. N. Chapman, Nano Lett. 8, 310 (2008).

[20] Y. Nishino, Y. Takahashi, N. Imamoto, T. Ishikawa, and K. Maeshima, Phys. Rev. Lett. 102, 018101 (2009).

[21] M. M. Seibert et al., Nature (London) 470, 78 (2011).

[22] H. N. Chapman et al., Nature (London) 470, 73 (2011).

[23] R. Koopmann et al., Nature Meth. 9, 259 (2012).

[24] A. Barty et al., Nature Photon. 6, 35 (2012).

[25] L. C. Johansson et al., Nature Meth. 9, 263 (2012).

[26] R. Neutze, R. Wouts, D. van der Spoel, E. Weckert, and J. Hajdu, Nature (London) 406, 752 (2000).

[27] Z. Jurek, G. Faigel, and M. Tegze, Eur. Phys. J. D 29, 217 (2004).

[28] T. Kai, Phys. Rev. A 81, 023201 (2010).

[29] S. P. Hau-Riege, R. A. London, and A. Szoke, Phys. Rev. E 69, 051906 (2004).

[30] S. P. Hau-Riege, Phys. Rev. A 76, 042511 (2007).

[31] C. Gnodtke, U. Saalmann, and J.-M. Rost, New J. Phys 13, 013028 (2011).

[32] S.-K. Son, L. Young, and R. Santra, Phys. Rev. A 83, 033402 (2011).
[33] S. Patil and Y. Varshni, Adv. Quantum Chem. 57, 1 (2009).

[34] A. Sil, S. Canuto, and P. Mukherjee, Adv. Quantum Chem. 58, 115 (2009).

[35] J. C. Slater, Phys. Rev. 81, 385 (1951).

[36] F. Herman and S. Skillman, Atomic Structure Calculations (Prentice-Hall, Englewood Cliffs, NJ, 1963).

[37] J. Stewart and K. Pyatt, Astrophys. J. 144, 1203 (1966).

[38] P. Hilse, M. Moll, M. Schlanges, and T. Bornath, Laser Phys. 19, 428 (2009).

[39] A. V. Gets and V. P. Krainov, J. Phys. B 39, 1787 (2006).

[40] S. Bhattacharyya, A. N. Sil, S. Fritzsche, and P. K. Mukherjee, Eur. Phys. J. D 46, 1 (2008).

[41] B. Saha, P. K. Mukherjee, and G. H. F. Diercksen, Astron. Astrophys. 396, 337 (2002).

[42] J. Saha, T. Mukherjee, P. Mukherjee, and B. Fricke, Eur. Phys. J. D 62, 205 (2011).

[43] P. K. Mukherjee, J. Karwowski, and G. H. Diercksen, Chem. Phys. Lett. 363, 323 (2002).

[44] B. Saha, T. Mukherjee, P. Mukherjee, and G. Diercksen, Theor. Chem. Acc. 108, 305 (2002).

[45] M. Das, R. K. Chaudhuri, S. Chattopadhyay, U. S. Mahapatra, and P. K. Mukherjee, J. Phys. B 44, 165701 (2011).

[46] M. Das, M. Das, R. K. Chaudhuri, and S. Chattopadhyay, Phys. Rev. A 85, 042506 (2012).

[47] M. S. Murillo and J. C. Weisheit, Phys. Rep. 302, 1 (1998).

[48] M. Moll, P. Hilse, M. Schlanges, T. Bornath, and V. P. Krainov, J. Phys. B 43, 135103 (2010).

[49] M. Moll, T. Bornath, M. Schlanges, and V. P. Krainov, Phys. Plasmas 19, 033303 (2012).

[50] Z. Jurek and G. Faigel, Eur. Phys. J. D 50, 35 (2008).

[51] N. Ashcroft and N. Mermin, Solid State Physics (Harcourt, Brace, New York, 1976).

[52] J. E. Robinson, F. Bassani, R. S. Knox, and J. R. Schrieffer, Phys. Rev. Lett. 9, 215 (1962).

[53] L. M. Sachs, Acta Crystallogr. 22, 931 (1967).

[54] G. B. Arfken and H. J.Weber, Mathematical Methods for Physicists, 5th ed. (Harcourt/Academic, San Diego, 2001).

[55] A. H. Boschitsch, M. O. Fenley, and W. K. Olson, J. Comput. Phys. 151, 212 (1999).

[56] J. P. Hansen and I. R. McDonald, Theory of Simple Liquids (Academic, London, 1986).

[57] A. A. Broyles, J. Chem. Phys. 35, 493 (1961).

[58] A. A. Broyles, J. Chem. Phys. 34, 1068 (1961).

[59] C. Z. Wang, K. M. Ho, and C. T. Chan, Phys. Rev. B 47, 14835 (1993).

[60] B. Held and P. Pignolet, J. Phys. France 47, 437 (1986).

[61] Z. Jurek, R. Thiele, B. Ziaja, and R. Santra, arXiv:1205.1393 (2012) [Phys. Rev. E (to be published)]. 